\title{
Observation of neutrinos with JEM-EUSO: an updated view
}

\author{
Elias Iwotschkin, Andrea Santangelo*, Alejandro Guzman, Thomas Mernik, Kenji \\ Shinozaki, Jörg Bayer \\ Institut für Astronomie und Astrophysik, Eberhard Karls Universität Tübingen, Germany \\ E-mail: iwotschkineastro.uni-tuebingen.de
}

\section{Gustavo Medina-Tanco}

Instituto de Ciencias Nucleares, Universidad Nacional Autonoma de Mexico, Mexico

\section{Angela V. Olinto}

Enrico Fermi Institute, University of Chicago, USA

\section{Lawrence Wiencke}

Department of Physics, Colorado School of Mines, USA

for the JEM-EUSO Collaboration

\begin{abstract}
Similarly to extreme energy cosmic rays (EECRs), neutrinos at energies exceeding $5 \times 10^{19} \mathrm{eV}$ are expected to interact in the Earth's atmosphere and create extensive air showers. The JEM-EUSO mission, developed to be hosted onboard the JEM module of the International Space Station, aims at detecting these extensive air showers from space by means of the fluorescent and diffusively reflected Cherenkov light they produce. In the present paper we investigate the capability of JEM-EUSO to trigger and detect neutrino induced events in the H-II Transfer Vehicle (HTV) configuration. We also discuss the status of their reconstruction feasibility considering JEMEUSO's most recent design, which will be launched with Falcon 9 and delivered to the ISS by the SpaceX Dragon free-flying spacecraft.
\end{abstract}

The 34th International Cosmic Ray Conference,

30 July- 6 August, 2015

The Hague, The Netherlands

\footnotetext{
*Speaker.
} 


\section{Introduction}

Over 50 years after the discovery of EECRs in the course of the Volcano Ranch experiment, the origin of these mysterious particles is still unknown. The main reason behind this fact is their extremely low flux at the highest energies which is thought to additionally suffer from the GreisenZatsepin-Kuz'min (GZK) cut-off at some $5 \times 10^{19} \mathrm{eV}[1,2]$. Here, the observation of neutrino primaries could be of great value.

Firstly, since UHE cosmic rays and photons are expected to lose energy via interactions with background radiation (such as the cosmic microwave background), the solely weakly interacting neutrinos could be the only primaries able to preserve their energy over cosmological distances. Secondly, because of their lack of electric charge neutrinos are not deflected by magnetic fields and point toward the direction of their origin. Furthermore, their huge interaction lengths (a few $\times 10^{7} \mathrm{~g} \mathrm{~cm}^{-2}$ above GZK cut-off energies) imply that they might come directly from the very core of their generation site, ignoring the surrounding matter, and carrying information on the cosmic particle generator itself.

In this paper we address the detection of such neutrino primaries by utilising the HTV design of the JEM-EUSO space-borne air shower detector, which we shall refer to as JE-HTV. Subsequently we present the reconstruction capability of such neutrino events in the Dragon spacecraft design of the JEM-EUSO observatory, abbreviated as JE-Dragon.

\section{The JEM-EUSO Mission}
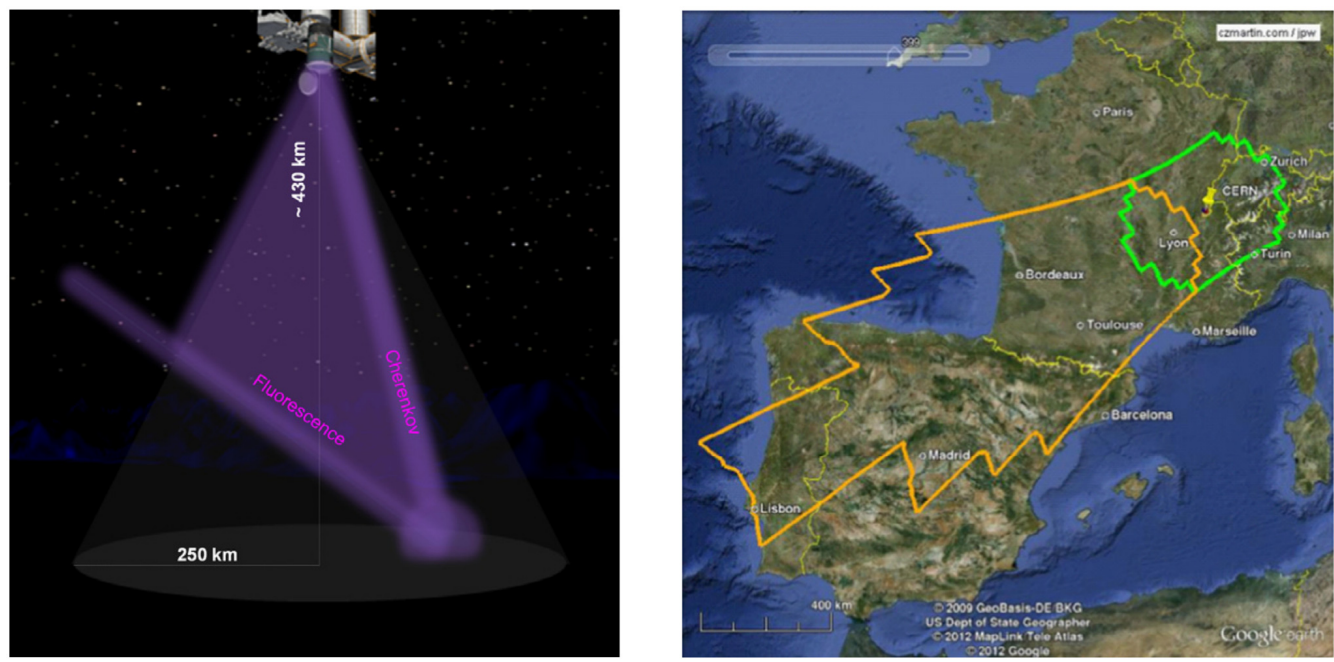

Figure 1: Left: The JEM-EUSO detection principle. [3] Right: Projection of the detection area onto Southwestern Europe in nadir (green) and tilted (orange) mode [4].

JEM-EUSO is short for Extreme Universe Space Observatory onboard the Japanese Experimental Module of the International Space Station (ISS). It is a space-based observatory which is being developed to search the Earth's atmosphere for fluorescence and Cherenkov photons in the range of 290-430 $\mathrm{nm}$ generated by extensive air showers. The detecting method is depicted on the left hand side of Fig. 1. 
The main scientific goals of the JEM-EUSO mission are the identification of sources of ultrahigh energy (UHE) particles, the measurement of the energy spectra of these individual sources, and the disclosure of the UHECR spectrum beyond $5 \times 10^{19} \mathrm{eV}$ which seems to experience a decrease due to the GZK effect. The discovery of neutrinos of extreme energies is one of the exploratory goals and the motivation for this paper. Reconstructing their energy could clarify if new types of sources are needed.

Such a space-borne instrument brings the advantage of a super wide field of view (FoV), $60^{\circ}$ in the case of JEM-EUSO, and a $4 \pi$ coverage of the celestial sphere. A special feature of the JEMEUSO concept is the possibility to tilt the telescope thereby increasing the observed area from $2 \times 10^{5} \mathrm{~km}^{2}$ in nadir mode to $7 \times 10^{5} \mathrm{~km}^{2}$ in tilted mode [4]. Two corresponding observation areas are shown on the right of Fig. 1.

The telescope consists of an optical system involving three Fresnel lenses, a $4.5 \mathrm{~m}^{2}$ focal surface (FS) detector arranged into photo-detection modules (PDMs) - groups of multi-anode photomultipliers (MA-PMTs) - with a total resolution of over 300000 px, and a sophisticated electronics design to reduce the amount of recorded data. The telescope's main design feature is a circular shape with two parallel side-cuts - resulting in a $2.65 \mathrm{~m}$ FS diameter, introduced to match JAXA's H-II Transfer Vehicle design.

\subsection{The Dragon Option}
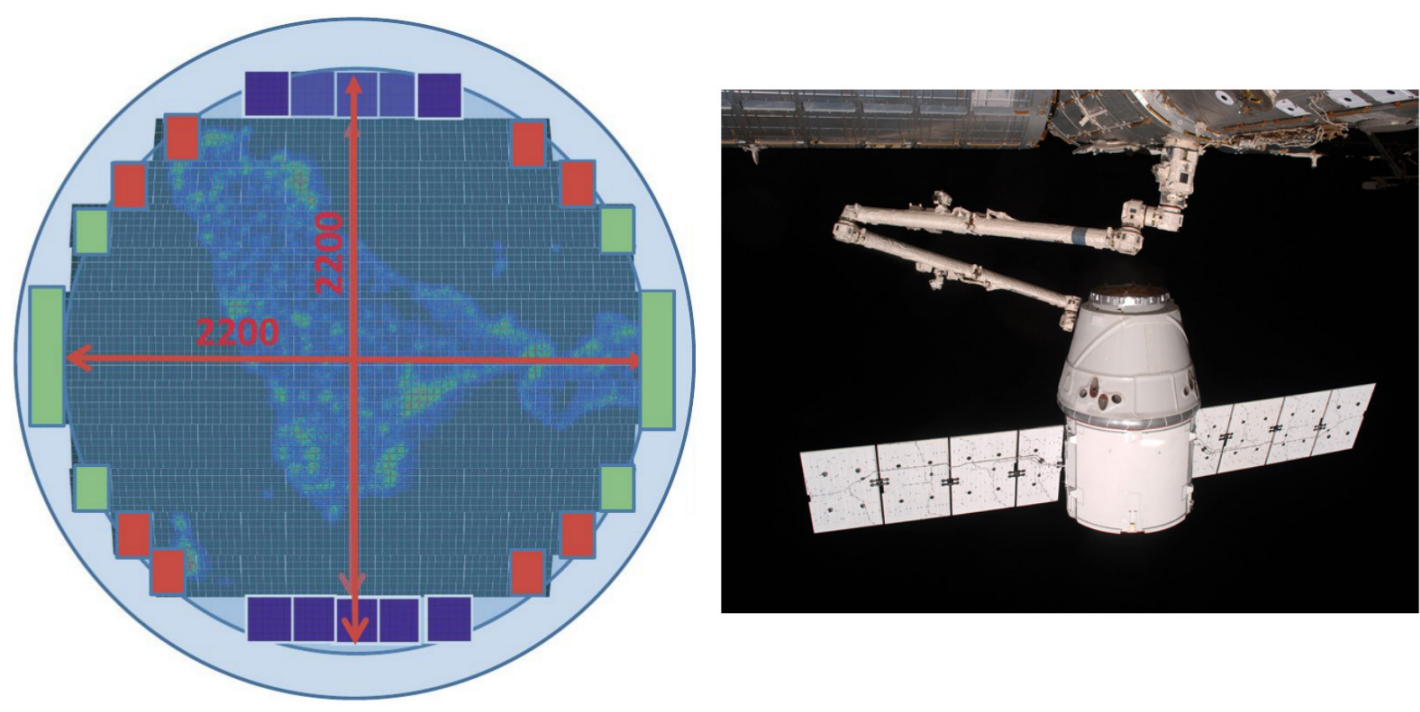

Figure 2: Left: Restructuring the FS from JE-HTV to JE-Dragon [8]. The green PDMs are rearranged into the blue areas, the red PDMs are removed. Right: A photograph of the Dragon spacecraft approaching the ISS [7].

With the development of the Dragon vessel by SpaceX in the recent past, an alternative transport option for the JEM-EUSO observatory has emerged which was studied in [5]. Dragon is a free-flying spacecraft that is launched via SpaceX's Falcon 9 rocket. It is the first commercial vessel to transport cargo to the ISS [6] and is shown on the right hand side of Fig. 2. With its total payload mass of $6 \mathrm{t}$ it can easily accommodate the JEM-EUSO instrument with a mass of under 2 $t$ in its unpressurised trunk. 
However, the accommodation of the observatory would require some modifications. Among the most important ones is a circular instrument design with a $2.5 \mathrm{~m}$ FS diameter as opposed to the side-cut one, whereby the aperture would be preserved, the stability increased and the weight reduced. The differences of the two FS designs are highlighted on the left side of Fig. 2. Furthermore, the attachment of the telescope by its heaviest part, the FS, allows for a telescopic frame of less mass [5].

\section{Simulating Neutrino-induced Showers}

To determine the primary particle that caused an EAS, the first interaction point, $X_{F I}$, and the shower maximum, $X_{\text {Max }}$, the point at which the shower holds the most particles, play key roles. Both are measured in $\mathrm{g} / \mathrm{cm}^{2}$ and are situated for a neutrino primary deeper in the atmosphere, although in this case $X_{\text {Max }}$ may not always exist.

For the assessment of the capability of the JEM-EUSO instrument to trigger and detect neutrinoinitiated showers, a combination of a few programs is applied to generate such events.

The first interaction of a neutrino with an atmospheric nucleon is done in an event generator called PYTHIA [9]. In this step, $10000 v_{\mathrm{e}}+\mathrm{n}$ events of the charged current type for each of the energies $10^{19.7} \mathrm{eV}, 10^{19.8} \mathrm{eV}, 10^{19.9} \mathrm{eV}$, and $10^{20} \mathrm{eV}$ are generated following the CT10 [10] parton distribution functions.
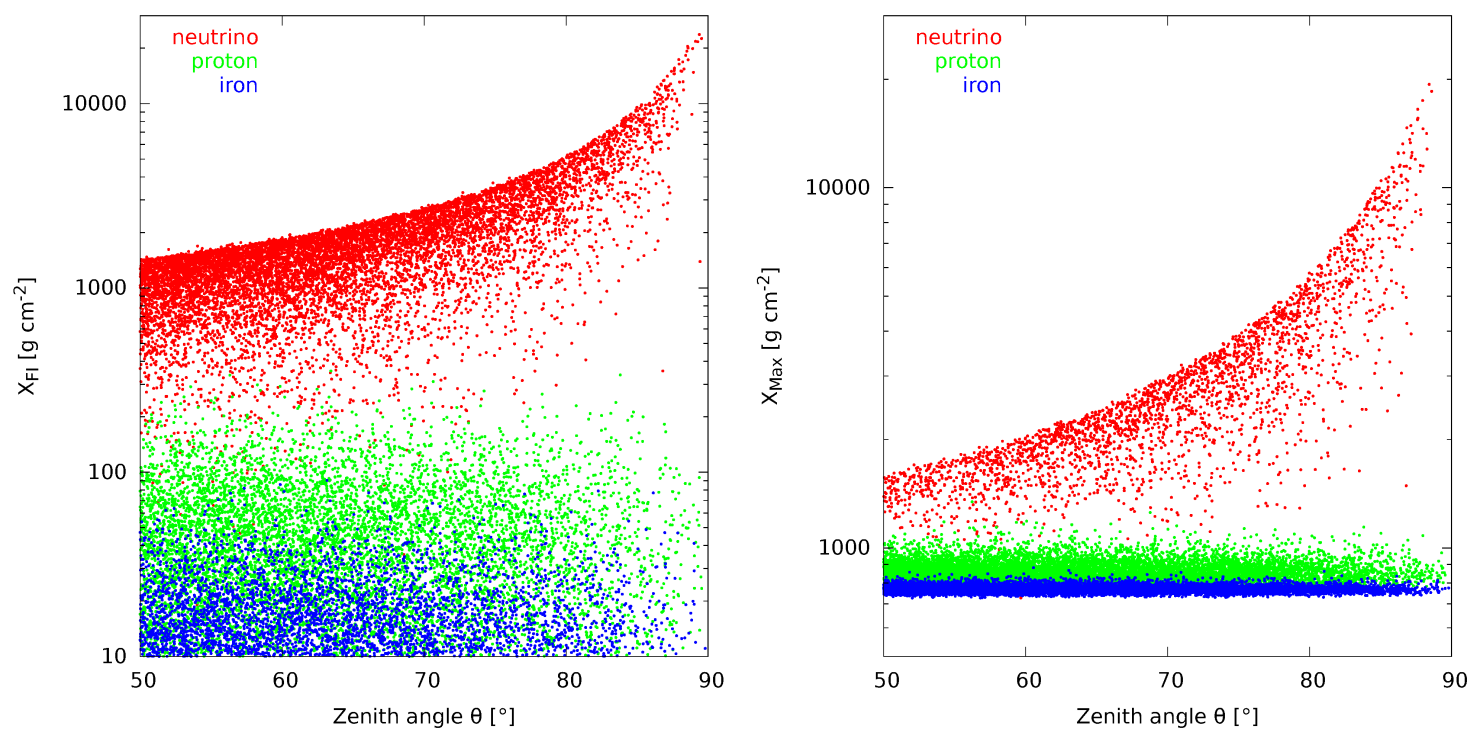

Figure 3: Presented are plots of first interaction (left) and shower maximum (right) slant depths against the zenith angles of neutrino, proton, and Fe-nucleus primaries. All events are simulated with CONEX and have an energy of $10^{20}$ $\mathrm{eV}$. Events in both plots are taken from the same samples. Note that while every neutrino shower has a first interaction point, only a subsample of these showers exhibits a shower maximum.

The resulting particle list is processed by the CONEX software [11]. Here, the extensive air shower development is treated in a hybrid method utilising the Monte Carlo approach and so called cascade equations. 
The showers' zenith angle, $\theta$, is following a $\sin (2 \theta)$ distribution. Neutrino events are expected to be observed for high zenith angles, hence $\theta$ is placed in the range between $50^{\circ}$ and $90^{\circ}$. The azimuthal angles are distributed equally from $0^{\circ}$ to $360^{\circ}$.

The depth in the atmosphere of the first interaction, $X_{F I}$, as well as of the shower maximum, $X_{\text {Max }}$, is shown in Fig. 3 for the case of $10^{20} \mathrm{eV}$. In the same plots, the slant depth of the first interaction and shower maximum as experienced by protons and Fe-nuclei of the same energy is shown for comparison. As it becomes clear from the plots, these quantities can be used to distinguish the type of primary particle. The first interaction points are distributed following the $1-\exp \left(-X / \lambda_{v}\right)$ function, where $\lambda_{v}$ is the energy-dependent interaction length of a neutrino.

Finally, the propagation of fluorescence and Cherenkov photons as well as their detection by the JEM-EUSO instrument is done in a software package called ESAF (EUSO Simulation and Analysis Framework) [12]. In the JE-HTV case, assuming a symmetric detector, the shower cores are placed in a rectangular area confining a quadrant of the FoV with the side lengths of $260 \mathrm{~km}$ and $190 \mathrm{~km}$. In the JE-Dragon scenario, the shower cores are placed within a $250 \mathrm{~km}$ radius around the center of the $\mathrm{FoV}$, following a $\sin (\zeta)$ distribution, with $\zeta$ being the angle around the center of the Earth spanned between the center of the FoV and the shower core position.

\section{Trigger Efficiency and Angular Reconstruction}

A space-based mission brings many constraints among which we find the telemetry restrictions. For these to be met a sophisticated trigger system is utilised. In the case of JEM-EUSO signal tracking triggers are applied at two hardware levels. The detector's capacity to distinguish a shower's signal from the nearly uniformly distributed background photons is described by the trigger efficiency. It is defined as the ratio of triggering events to all events in a sample, multiplied by the ratio of injection to FoV areas

$$
\varepsilon_{\text {trig }}=\frac{N_{\text {trig }}}{N_{\text {samp }}} \cdot \frac{A_{\text {inj }}}{A_{\text {FoV }}}
$$
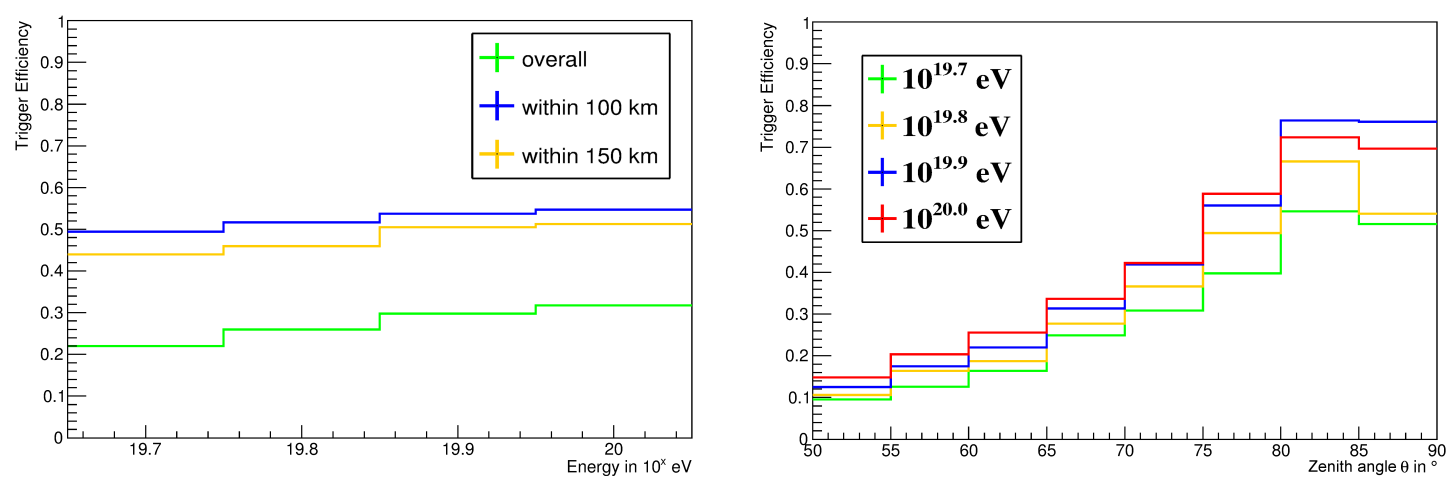

Figure 4: Both plots show trigger efficiencies for the JE-HTV detector [13]. Left: The trigger efficiency is plotted against primary energies; the histograms represent different subsets of the shower samples as shown in the legend. Right: The trigger efficiency is plotted versus the event zenith angle. Different histograms represent the four energies shown in the legend. 
For the four shower samples in the JE-HTV scenario we obtain trigger efficiencies shown in Fig. 4. In the chosen energy interval one can observe an increase with rising energy presented in the left plot of the same figure. Higher energy primaries naturally cause a particle-richer air shower resulting in more fluorescence photons and a higher trigger probability. Similarly, a more inclined neutrino can have a longer atmospheric path to develop an EAS and thus becomes more probable for detection than a less inclined one. A corresponding plot is presented on the right of Fig. 4. We observe a steep increase toward zenith angles beyond $80^{\circ}$ where the trigger efficiencies exceed 50 $\%$ even for the lowest simulated energy, i.e. $5 \times 10^{19} \mathrm{eV}$. Analogous plots for the JE-Dragon design are being processed and will be available in the near future.

After the simulations are concluded, ESAF allows the user to reconstruct the simulated events. For the angular reconstruction a couple of algorithms are implemented. In the following graphs they are denoted as AA1, AA2, NE1, NE2, and AE1 and are further described in [14, 15]. The angle between the true incoming position of the primary and the reconstructed one is called the separation angle $\gamma$. For the JE-Dragon configuration it is plotted against the zenith angle $\theta$ for all four samples combined in Fig. 5. One can observe that for the bulk of inclinations and algorithms $\gamma$ is situated between $2^{\circ}$ and $4^{\circ}$.

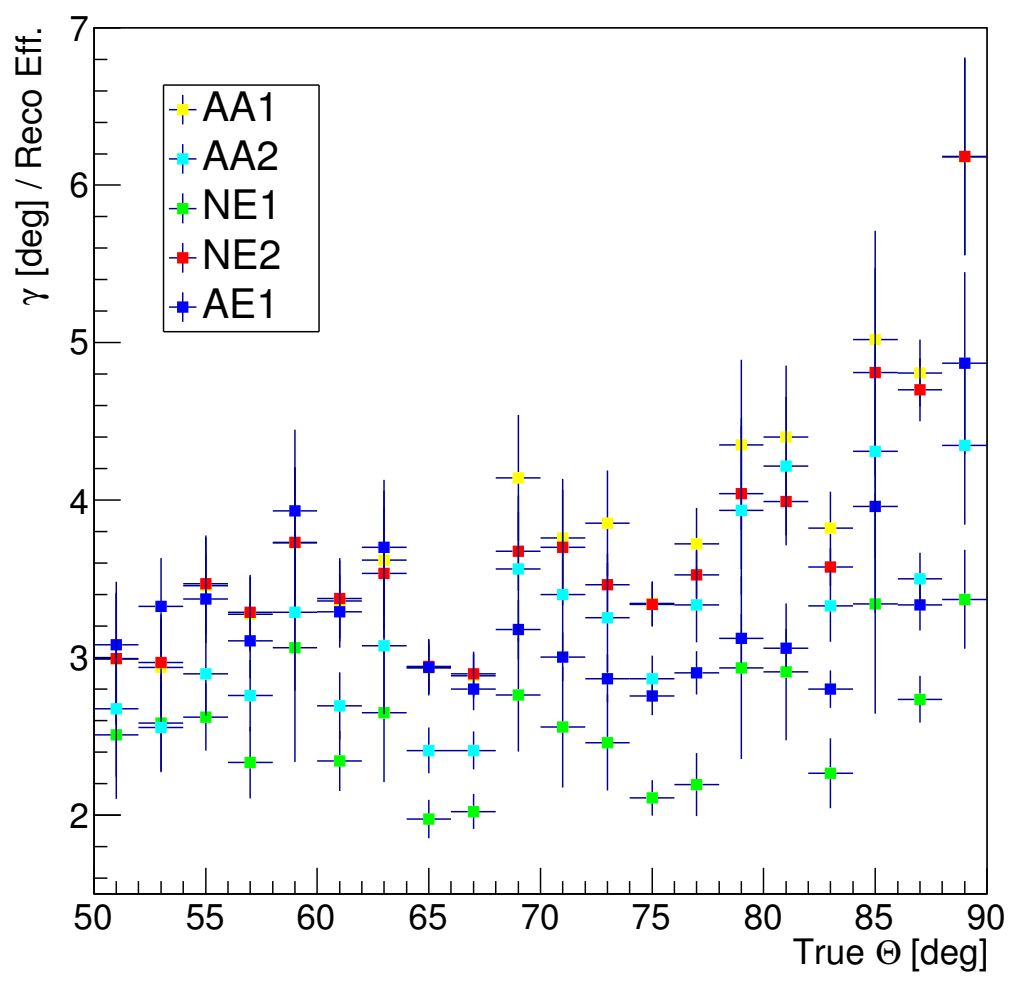

Figure 5: Shown is the angular difference, $\gamma$, between reconstructed and real directions, obtained with five different algorithms: AA1, AA2, NE1, NE2, AE1. For more information on angular reconstruction algorithms see [14, 15].

Disentangling the zenith and azimuth angles, we obtain the differences between the reconstructed and true angles as shown in Fig. 6. The left side depicts the differences in zenith, $\theta_{\text {reco }}-\theta$, 
the right in azimuth angles, $\phi_{\text {reco }}-\phi$. One can see that while the azimuthal differences are rather small and on average around zero, the zenith angles are underestimated with increasing inclinations by the reconstructing algorithms. This is a systematic feature inherent to proton reconstruction, as well, and will be analysed in the near future.
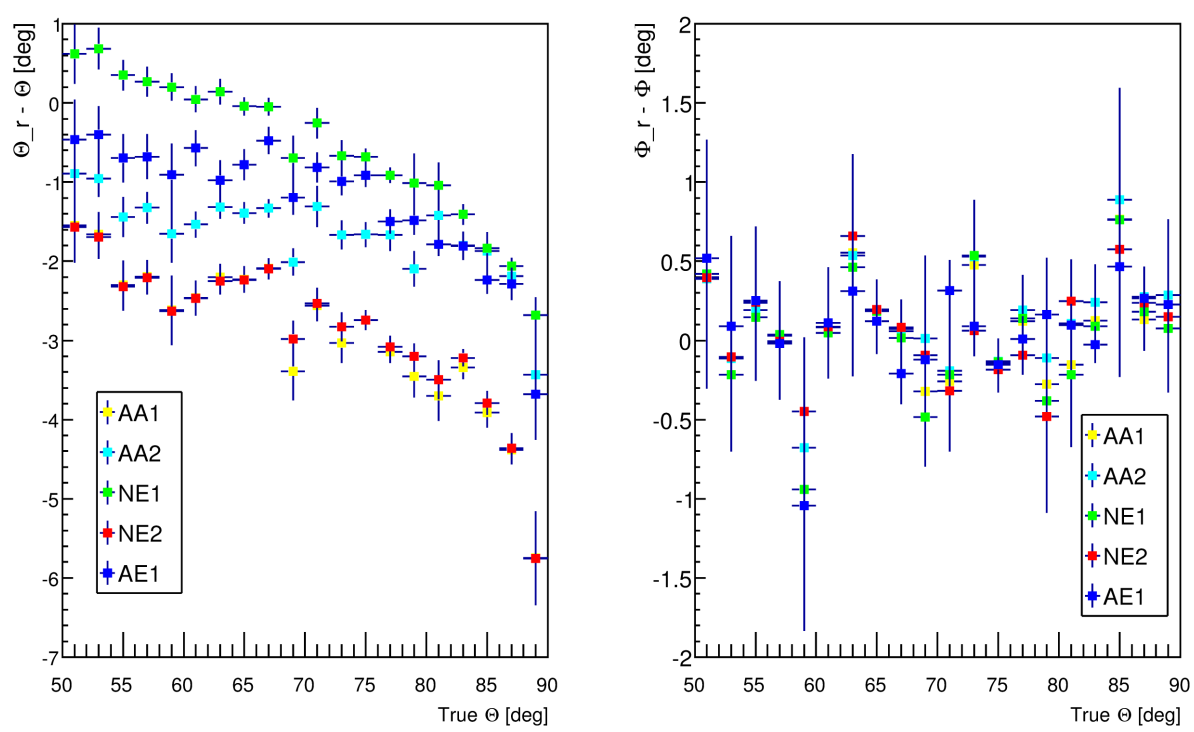

Figure 6: Shown are the differences between true and reconstructed zenith (left) and azimuthal (right) angles. The same five algorithms as in the plot above were applied.

Concerning the energy reconstruction, ESAF is optimised for the parametrised proton case. A reasonable reconstruction of neutrino event energies is not feasible at the moment. However, a preliminary attempt to reconstruct proton-, Fe-nucleus-, and photon-initiated events was done in [16].

\section{Conclusion and Outlook}

This paper is concerned with the simulation of samples of neutrino-initiated events in the scope of the JEM-EUSO mission. We presented the JE-HTV trigger efficiencies for neutrino events. For beyond-GZK energies and sampled over zenith angles $>50^{\circ}$, we observe efficiencies well above $20 \%$. For highly inclined events $\left(>80^{\circ}\right)$ these efficiencies easily surpass the $50 \%$ line.

Additionally, angular reconstructions of triggering events for the JE-Dragon setup were performed, over $80 \%$ of these events could be reconstructed. A promising picture is further drawn by the relatively small difference of less than $3^{\circ}$ in the direction separation angle $\gamma$ for most inclinations.

Acknowledgment: This work was partially supported under the ESA Topical Team Contract No. 4000103396, by the Bundesministerium für Wirtschaft und Technologie through the Deutsches Zentrum für Luft- und Raumfahrt e.V. (DLR) under the grant number FKZ 50 QT 1101, by PAPIIT-UNAM/CONACyT, by Basic Science Interdisciplinary Research Projects of RIKEN and JSPS KAKENHI Grant (22340063, 23340081, and 24244042), by the Italian Ministry 
of Foreign Affairs, General Direction for the Cultural Promotion and Cooperation, by the 'Helmholtz Alliance for Astroparticle Physics HAP' funded by the Initiative and Networking Fund of the Helmholtz Association, Germany, and by Slovak Academy of Sciences MVTS JEM-EUSO as well as VEGA grant agency project 2/0076/13. Russia is supported by the Russian Foundation for Basic Research Grant No 13-02-12175-ofi-m. The Spanish Consortium involved in the JEM-EUSO Space Mission is funded by MICINN \& MINECO under the Space Program projects: AYA200906037-E/AYA, AYA-ESP2010-19082, AYA-ESP2011-29489-C03, AYA-ESP2012-39115-C03, AYA-ESP2013-47816C4, MINECO/FEDER-UNAH13-4E-2741, CSD2009-00064 (Consolider MULTIDARK) and by Comunidad de Madrid (CAM) under projects S2009/ESP-1496 \& S2013/ICE-2822.

\section{References}

[1] K. Greisen, End to the Cosmic Ray Spectrum?, Physical Review Letters 16 (1966) 748

[2] G.T. Zatsepin, V.A. Kuz'min, Upper Limit of the Spectrum of Cosmic Rays, Soviet Journal of Experimental and Theoretical Physics Letters 4 (1966) 78

[3] A. Santangelo et al., Space Based Studies of UHE Neutrinos, Progress in Particle and Nuclear Physics 64 (2010) 366

[4] T. Ebisuzaki, G. Medina-Tanco, A. Santangelo - JEM-EUSO Collaboration, The JEM-EUSO Mission, Advances in Space Research 53 (2014) 1499

[5] J.H. Adams Jr., R.M. Young, A.V. Olinto - JEM-EUSO Collaboration, JEM-EUSO Design for Accommodation on the SpaceX Dragon Spacecraft, Proceedings of the 33rd International Cosmic Ray Conference (2013)

[6] Online: http://www.spacex.com/dragon, accessed: 20.06.2015

[7] Online: http://www.spacex.com/media-gallery/detail/1665/210, accessed: 20.06.2015

[8] M. Casolino, JEM-EUSO internal presentation (2013)

[9] T. Sjöstrand, S. Mrenna, P.Z. Skands, PYTHIA 6.4 Physics and Manual, Journal of High Energy Physics 5 (2006) 26

[10] H.L. Lai et al., New Parton Distributions for Collider Physics, Physical Review D 82 (2010) 074024

[11] T. Bergmann et al., One-dimensional Hybrid Approach to Extensive Air Shower Simulation, Astroparticle Physics 26 (2007) 420

T. Pierog et al., First Results of Fast One-dimensional Hybrid Simulation of EAS Using CONEX, Nuclear Physics Proceedings Supplements 151 (2006) 159

[12] A. Thea et al., The EUSO Simulation and Analysis Framework, Proceedings of the 29th International Cosmic Ray Conference 9 (2005) 133

C. Berat et al., Full Simulation of Space-based Extensive Air Showers Detectors with ESAF, Astroparticle Physics 33 (2010) 221

[13] E. Iwotschkin, Space Based Observation of Ultra-high Energy Neutrinos with EUSO, Master thesis, Universität Tübingen (2015)

[14] T. Mernik, The Expected Angular Resolution of the JEM-EUSO Mission, PhD thesis, Universität Tübingen (2014)

[15] S. Biktemerova, A. Guzman, T. Mernik, Performances of JEM-EUSO: angular reconstruction - The JEM-EUSO Collaboration, Experimental Astronomy (2014)

[16] A. Guzman et al., JEM-EUSO Observational Capabilities for Different UHE Primaries, Proceedings of the 34th International Cosmic Ray Conference ID 0570 (2015) 